\section{TRANSMISSION OF POLARISED LIGHT THROUGH UNIAXAL CRYSTALS}

THE appearances presented by the transmission of polarised light through crystals, have long been known as the most magnificent in Optics. It is our intention in this paper to give an account of the more recent observations which have been made respecting the phenomena exhibited by uniaxal crystals, accompanied by such an explanation as will, we hope, render them intelligible to persons very slightly acquainted with science. We shall therefore avoid as much as we can the use of technical terms, and assume as little as possible to be previously known to the reader.

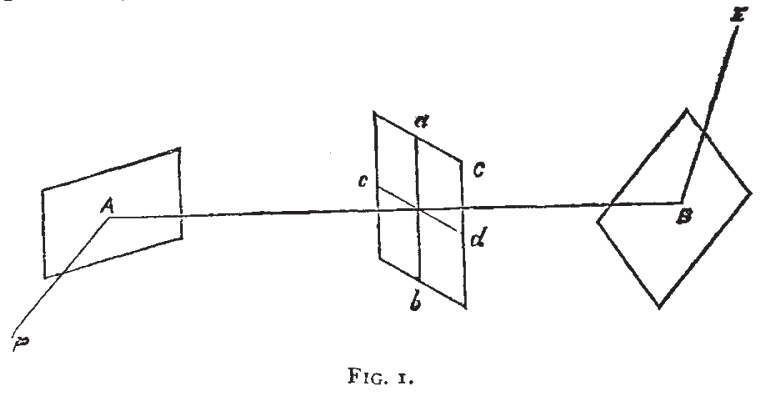

Common light consists of undulations of the supposed xtherial medium, in which the vibrations of each particle are perpendicular to the direction of the wave's motion, and in every conceivable direction which this condition adinits.

Suppose now a ray of sunlight reflected from a plate of unsilvered glass. The principle of the composition and resolution of forces plainly enables us to regard it as made up of two sets of vibrations, one in the plane of reflection, and the other perpendicular to it. Now for reasons which cannot be explained to a reader unacquainted with mathematics, * it is found that the vibrations of the reflected ray in the plane of reflection, when the angle of

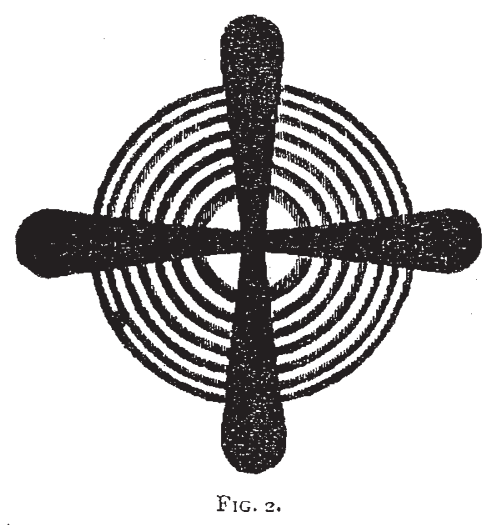

reflection is equal to $56^{\circ} 30^{\prime}$, wholly disappear, so that the reffected ray consists entirely of vibrations perpendicular to the plane of reflection, and is said to be polarised. The reader will carefully remember the distinction between common light and polarised light. Common light is light in which the vibrations occur in every conceivable plane passing through the ray; polarised light is light in which the vibrations occur in only one plane of fixed inclination passing through the ray.

Now let a ray of sunlight fall upon a plate of unsilvered glass at an angle of about $56^{\circ}$, let the reflected ray be received by another glass plate at the same angle, and be

* The mathematical reader is referred to Green's "Menoir on the Reflection of Liglit," Cambridge Pinilosophical Transactions, vol. vii. thence reflected to the eye. Suppose the position of the mirror to be such that the planes of first and second incidence and reflection are perpendicular to each other. Then remembering what we have just stated, we see that the vibrations of the ray reflected from the first mirror lie altogether in the plane of second incidence. These vibrations, therefore, will all be destroyed by the second reflection, therefore nothing but a dark spot will be perceptible to the eye.

If now the position of the second mirror be shifted, so that the planes of first and second incidence coincide with each other, while the angle of second incidence remains unaltered, the vibrations of the incident ray will all lie perpendicular to the plane of second incidence, and therefore a bright spot will be visible to the eye.

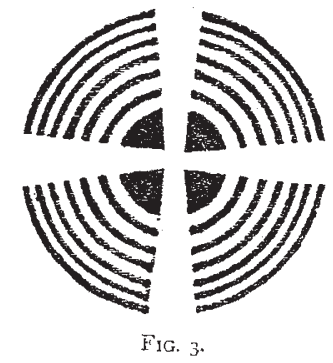

Let now the second mirror be gradually turned round an axis situated in the direction of the ray incident upon it from the first position into the second, then the angle of second incidence will remain unaltered during the revolution, while the plane of second incidence will revolve with the mirror, and, as might be expected, light will begin to be visible to the eye and continually increase, till it attains its maximum in the second position of the mirror. It is usual to call the first mirror the polariser, the second the analyser.

Let a plate cut from a transparent crystal be interposed between the polariser and analyser thus arranged, luminous

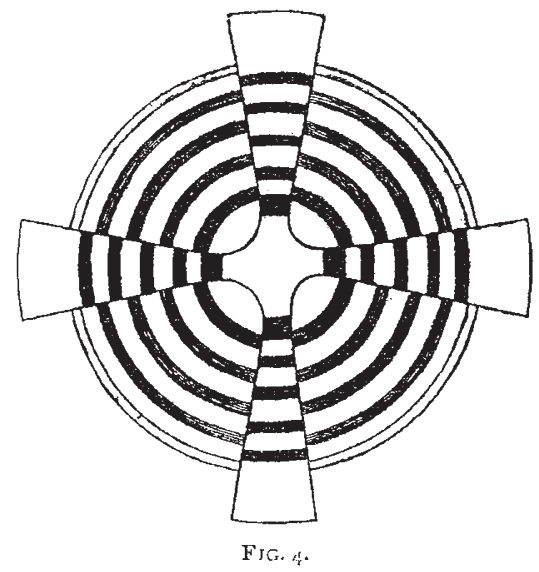

curves of varying colours intersected by dark bands will be visible to the eye. Before describing these gorgeous appearances, we must say a few words on the modification light undergoes in passing through a crystal.

When a ray of light passes through a crystal, it is in general divided into two, so that if a plate of crystal be interposed between the eye and a luminous point, the point will appear doubled. But in all crystals there is one, and in some two fixed directions in which no bifurcation of the ray takes place. If we look along one of these lines, called the optic axes, we shall see only one image of the object of vision. Crystals with one axis are called uniaxal crystals; those with two axes are called biaxal 
crystals, and in this paper I shall confine myself to the former.

We observe that a plane drawn through the direction of the ray or pencil and the axis of the crystal is called a principal plane. It is found that one of the two rays into which the incident ray is divided is refracted according to the ordinary law, and is therefore called the ordinary ray, the other according to a more complicated law, and is called the extraordinary ray. But we especially remark that the vibrations constituting the ordinary ray lie wholly perpendicular to the principal plane of the crystal, those of the extraordinary ray parallel to the principal plane. Consequently both rays will consist of polarised light, according to the definition we have just given of polarisation, and this polarised light will arise from the vibrations of the incident ray being resolved perpendicular and parallel to the principal plane.

Of all uniaxal crystals the best known is Iceland spar, which possesses the power of double refraction in an eminent degree. It occurs in the form of a rhombohedron, and the axis of the crystal corresponds with the shortest diagonal.

Now suppose a plate of this crystal, cut perpendicular to its axis, is interposed between the polarising and analysing plates, when the first and second planes of incidence are perpendicular to each other.

The colour of light depends on the length of the wave. The length of a wave of violet light is 0000167 , and that of a wave of red light 0000266 of an inch. The wavelengths of the other colours lie between these. White light is a compound of all colours, and therefore its vibrations are of all lengths lying between these extremes. It will therefore manitestly simplify the explanation we are going to submit to the reader, if we commence by assuming the light to be monochromatic, and its vibrations of the same length. Let a small pencil of monochromatic light, $P$ (Fig. $I$ ), be reflected from a plate of glass, $A$, at an angle of about $56^{\circ}$, pass through the crystal $C$, and then be reflected a second time from the plate $B$ at an angle of about $56^{\circ}$ in the direction $\mathrm{B} \mathrm{E}$. The plane $\mathrm{PA} \mathrm{B}$ is supposed to be perpendicular to the plane of the paper, the plane $A B E$ to coincide with it. The crystal is supposed to be bounded by parallel surfaces perpendicular to its axis, and in a position perpendicular to the line $\mathrm{A} B$. Let $a b, c d$, be two straight lines drawn in the crystal, one in the plane of the paper and the other perpendicular to it. The eye is supposed to be placed at E.

The vibrations of light proceeding from $A$ will all be parallel to the plane of the paper. In general, each ray as it enters the crystal will be resolved into two, and thus will arise two sets of waves which will interfere with each other. Hence a succession of dark and bright curves will be visible to an eye placed at $\mathrm{E}$. Moreover, as the crystal is symmetrical round $\mathrm{A} \mathrm{B}$, it may be expected that these curves will be circular. It may also be expected that the diameters of the bright circles will depend on the length of the wave, and therefore on the colour of the light.

We have already defined the principal plane of the crystal for a given ray, to be the plane passing through the ray and through the axis of the crystal, which in this case coincides with A B. As therefore the vibrations of the rays incident on the crystal are all parallel to the plane of the paper, those which enter the crystal along $c d$ are all perpendicular to a principal plane of the crystal, and those which enter it along $a b$ are all parallel to a principal plane. Therefore every ray which enters the crystal along $a b$ and $c d$, will be transmitted to the second plate unresolved, and unchanged, and will therefore be incapable of being reflected to an eye sitiate at $\mathrm{E}$. Consequently a dark cross will appear to the eye at $E$, corresponding to the lines $a b, c d$, intersecting the system of rings we have just described. Now suppose white light to be substituted for monochromatic light. White light is composed of light of all colours, and we have seen that the diameters of the bright rings are different for each colour. Consequently, instead of dark and bright rings, we shall have a series of coloured rings intersected by a dark cross. This phenomenon is shown in Fig. 2.

If the second plate be turned round an axis in the direction $A B$, till the first and second planes of reflection coincide, the reader who has followed this investigation will perceive that the dark cross intersecting the rings will be changed into a white cross. This bright cross is shown in Fig. 3 .

If the second plate is turned round the same axis into any position intermediate to those we have just described, the rings will be intersected by two crosses, inclined to each other at an angle equal to that between the planes of first and second reflection. There will be coloured arcs between the crosses, but those distances which give a maximum light for the arcs outside the crosses will give a minimum light for the arcs inside the crosses. This phenomenon is shown in Fig. 4. These are the appearances observed when the surfaces of the crystal plate are perpendicular to the axis of the crystal, and to the axis of the incident pencil. Dr. Ohm has investigated the phenomena which occur when the axis of the crystal is inclined at any angle to its surface, and the crystal itself is placed in any position between the polarising and analysing plates. His mathematical investigations will be found in the seventh volume of the "Munich Transactions," and the following are the principal results which he has obtained.

We shall suppose the light monochromatic. There will be a succession of dark and bright curves which are either parabolas, ellipses, or hyperbolas. For a given position of the crystal, the ellipses and hyperbolas are concentric; but the centres are never in the centre of vision, unless the axis of the crystal is either perpendicular or parallel to its surfaces. When the bright curves are parabolas, their vertices are equidistant from one another. When the bright curves are ellipses or hyperbolas, the difference of the squares of the semiaxes of two consecutive curves is a constant quantity. We have seen that when the axis of the crystal is perpendicular to its faces, the bright curves are circles. As we cut the plates so that the axis may be inclined at angles more and more acute to the surfaces, the bright circles become ellipses, which elongate continually till they become parabolas.

Suppose two plates of crystal, with parallel surfaces of equal thickness, and with their axes inclined at the same angle to the parallel surfaces, be placed in contact, so that their axes may lie in the same plane, but not in the same straight line, and then introduced between the polarising and analysing plates, a succession of dark and bright ellipses and hyperbolas, with their centres in the centre of vision, will be seen. It was this experiment which led Dr. Ohm to the results we have endeavoured to lay before the reader.

W. H. L. RUSSELL

\section{NOTES}

Twelve months ago the Erdington Orphanage, founded, built, and endowed solely by Mr. Josiah Mason, at a cost of nearly 250,000l., was opened at Birmingham. Mr. Mason, exactly following the example of $\mathrm{Mr}$. Peabody, has now in contemplation another public work of even greater ultimate importance, namely, a college and schools for scientific and technical instruction, open to all classes, and if the hopes of the founder should be realised, capable of expansion into one of the noblest institutions in the kingdom. As yet the plan is only broadly formed, and some time must elapse before it can be carried into effect ; but the Birmingham Daily Post states that a beginning has been made, and that for the purpose above mentioned, Mr. Mason has agreed to buy a large block of land in Edmund Street, exactly facing Ratcliff Place, between the Town Hall and 